\title{
Implementation of a Tracheostomy Protocol During the COVID-19 Pandemic
}

\author{
Brian Temple*, Michael Segal, Vijay A Singh, Daniel Galvin, Robert Kerr, Robert Zingale \\ Huntington Hospital - Northwell Health, Huntington, New York, USA \\ Email address: \\ templebc1@gmail.com (B. Temple) \\ ${ }^{*}$ Corresponding author \\ To cite this article: \\ Brian Temple, Michael Segal, Vijay A Singh, Daniel Galvin, Robert Kerr, Robert Zingale. Implementation of a Tracheostomy Protocol \\ During the COVID-19 Pandemic. International Journal of Cardiovascular and Thoracic Surgery. Vol. 6, No. 3, 2020, pp. 38-43. \\ doi: $10.11648 /$ j.ijcts.20200603.11
}

Received: June 19, 2020; Accepted: July 7, 2020; Published: July 17, 2020

\begin{abstract}
The coronavirus SARS-CoV-2 (COVID-19) pandemic has offered a unique set of challenges to the medical community often requiring prolonged treatment algorithms. The illness, afflicting more than 7.3 million people worldwide with estimates of 5-20\% requiring critical care, has become a burden on the healthcare community. These critically ill patients who acquire the severe form of the disease routinely require prolonged invasive mechanical ventilation. The questions then arise, "when and for whom does tracheostomy become indicated," and "how to safely perform a tracheostomy in this patient population." With consideration to aerosolization of the virus, we have derived and instituted a protocol at a community institution with aims of reducing provider risk while safely performing a tracheostomy. An open tracheostomy was performed at bedside, within a negative pressure intensive care unit (ICU) setting, utilizing a closed-circuit technique as described in this text. A total of 17 tracheostomies were performed employing the described technique. Minimal complications were noted throughout the study and no adverse oxygenation events were observed with an average total apneic time of 106 seconds. An acceptable mortality rate of $23 \%$ was observed given the lethal nature of this disease in ventilated, critically ill patients. No nosocomial transmission of the virus was documented for all team members. This protocol can be used to determine efficacy and safely execute a tracheostomy in COVID-19 patients. As information about COVID-19 continues to unfold, protocols for high risk procedures will need to fluidly evolve.
\end{abstract}

Keywords: COVID-19, Tracheostomy, Protocol, Indication

\section{Introduction}

At present, the COVID-19 pandemic has a confirmed 7.38 million cases and 415,000 deaths worldwide with an estimated 53,000 of cases requiring critical care [1]. In early studies approximately $9.8-15.2 \%$ of infected individuals have been found to require invasive mechanical ventilation [2]. Healthcare systems around the world have responded by increasing their critical care capacity and have therefore accumulated an abundance of chronically endotracheal intubated patients. Traditional ICU care offers a tracheostomy around intubation day 7-10 to provide a stable surgical airway, good pulmonary hygiene, shorter ICU/hospital stays and overall comfort to these patients [3]. With this influx of endotracheally intubated patients, with a highly transmissible pathogen, one must question the timing, efficacy, and proper execution of tracheostomy.

The novel coronavirus outbreak, shares a common route of infection due to airborne and fine droplet transmission via human-human contact and eventual entry via mucosal membranes and conjunctiva with the Severe Acute Respiratory Syndrome (SARS) and Middle East Respiratory Syndrome (MERS) outbreaks [4, 5]. Therefore, we sought out to implement similar protocols as during these epidemics. In the previous SARS/MERS outbreaks, there is very little literature regarding tracheostomy, mostly consisting of small case report studies and surgical protocol recommendations. Procedures such as endotracheal intubation, bronchoscopy, and tracheostomy are considered high risk procedures for providers due to their propensity for aerosolization of viruses. These studies recommend utilization of additional personal protective equipment and performance of an open 
tracheostomy in a negative pressure, ICU, bedside setting to reduce high viral load aerosolization. No long-term studies were undertaken to evaluate the efficacy and safety of those procedures however no immediate adverse events were identified and no healthcare workers contracted the virus postoperatively $[6,7,8,9]$. Seemingly, the low rates of tracheostomies performed in the aforementioned diseases are likely secondary to the potential of high provider risk and the determination of overall futility of surgical intervention.

With the onset of COVID-19 there have been many recommended protocols offered within the literature to safely perform high risk procedures with a corresponding absence of literature demonstrating implementation and execution of these techniques [10-13]. As our institution is located within the greater New York area we have been heavily burdened by this pandemic and may be among the first in the United States to undertake high risk procedures such as tracheostomy. We attempted to balance the risks involved to the healthcare workers while also addressing the ethical dilemma of which patient would be deemed acceptable for tracheostomy. We propose a thorough preoperative checklist that was formulated in a multidisciplinary manner to establish those who were believed to have optimal long-term viability. In similar fashion, we established a surgical protocol in performing a tracheostomy, while minimizing the aerosolization time period limiting the nosocomial transmission of this harrowing disease.

\section{Methods}

A multidisciplinary team of specialists was assembled in order to procure a safe tracheostomy protocol that would limit nosocomial transmission of COVID-19. The steps taken in creating this protocol are with the design of maintaining a closed circuit to reduce exposure to aerosolized virus. Tracheostomies were limited to 3 per day in order to minimize fatigue and instance of breach of protocol. The preoperative evaluation, indications, equipment needed, necessary personnel and protocol are outlined below.

\subsection{Indications}

Patients approved by our team for tracheostomy had to meet our indication criteria for tracheostomy (Table 1). Patients must be diagnosed COVID-19 positive via endotracheal nasal swab sent for Polymerase chain reaction (PCR) analysis and be endotracheally intubated for a minimum of 21 days. Ventilator settings must not exceed a fraction of inhaled oxygen $\left(\mathrm{FiO}_{2}\right)$ of $40 \%$ and a positive end expiratory pressure (PEEP) greater than $8 \mathrm{mmHg}$. A complete neurological exam was performed by a neurosurgeon to evaluate reasonable cognitive function. Acute phase reactants such as C-reactive Protein, Erythrocyte sedimentation rate and Procalcitonin levels must be observed to downtrend. Lastly, patients chosen to undergo tracheostomy revealed a promising prognosis and ideally minimal co-morbidities.

\subsection{Equipment}

A standardized checklist of necessary equipment was devised and made readily available by the operating room staff (Table 2). A negative pressure ICU bed setting was used in order to perform all tracheostomies. Standard, full, disposable personal protective equipment (PPE) consisting of N95 mask, eye protection (face shield or goggles), sterile operating room (OR) gowns and gloves, shoe covers and scrub caps were utilized. A standard open tracheostomy tray and Bovie electrocautery was brought from the OR to the ICU. Cuffed tracheostomy tubes ranging in size from 7-8 Shiley with extended length tracheostomy (XLT) options were available. A portable headlight and shoulder roll were vital in obtaining optimal visualization of tracheal dissection. A viral filter compatible with ventilator tubing was placed prior to ventilation after the procedure was completed. Numerous hemostatic agents were readily made available at bedside.

\subsection{Personnel}

A dedicated tracheostomy team of specialists was chosen who were preoperatively briefed on the details of the procedure. This team included the following personnel (Table 3): attending anesthesiologist, attending general/thoracic surgeon, chief surgical resident first assistant, surgical scrub nurse, a circulating registered nurse $(\mathrm{RN})$ and respiratory therapist. All procedures were performed by 2 senior Attending Surgeons with the same chief resident. The above individuals donned the appropriate PPE and were the only individuals allowed within the converted ICU operating room. A second circulating RN was on standby outside of the ICU operating room, in order to allocate unforeseen supplies in a fastidious manner.

\subsection{Procedure}

The dedicated tracheostomy team would conduct a preoperative brief of the procedure and don all necessary PPE prior to entering the negative pressure ICU room. A surgical time out was performed, confirming all personnel and equipment was available. The patient was placed on $\mathrm{FiO}_{2} \%$ of $100 \%$ and paralytic anesthesia administered. A shoulder roll was used for adequate exposure of the surgical field. The patient was prepped and draped in sterile fashion. Skin incision was made and trachea exposed via dissection with bovie electrocautery which was used at 40 Watts and decreased to 20 Watts once trachea visualized. Once the trachea was satisfactorily dissected, the anesthesia team was queued to turn off/hold the ventilator, deflate the endotracheal cuff and advance the endotracheal tube (ETT) approximately $2 \mathrm{~cm}$. The cuff was then re-inflated and the ventilator turned back. The time frame spent off the ventilator above was deemed apneic period 1 (AP1).

The patient was pre-oxygenated for approximately 30 seconds, thereafter the anesthesiologist would queue the surgical team to proceed. A tracheal hook was used to stabilize the trachea at the cricoid cartilage. The ventilator was then 
turned off/held, the patient was allowed to exhale and the endotracheal tube was clamped. The tracheotomy was then created and dilated. The endotracheal tube was withdrawn by the anesthesiologist under direct visualization of the surgical team. The trachea was then intubated with an appropriate sized tracheostomy tube. The tracheostomy cuff was over-inflated and tracheostomy connected to the ventilator with a viral filter.
Ventilation was then resumed. End tidal Carbon dioxide and adequate tidal volume administration were used to confirm positioning of the Tracheostomy. The second time frame spent off the ventilator above was deemed apneic period 2 (AP2). The skin incision was closed and the tracheostomy flange was secured with 2-0 silk sutures. The above tracheotomy protocol is summarized in Table 4.

Table 1. Indications for Tracheostomy.

\begin{tabular}{ll}
\hline 1 & COVID $19+$ \\
2 & Endotracheal intubation for $>21$ days \\
3 & Minimal Vent Settings: $\mathrm{FiO}_{2} \%<40 \%$, PEEP $<8 \mathrm{mmHg}$ \\
4 & Preoperative stable Neurologic Examination (Neurology/NeuroSurgical Specialist) \\
5 & Promising prognosis with minimal Co-morbidities \\
\hline
\end{tabular}

Table 2. Equipment Utilized for Tracheostomy.

\begin{tabular}{ll}
\hline 1 & Negative pressure ICU room. \\
2 & Standard Open Tracheostomy tray/ Head \& Neck Sterile drapes. \\
3 & Bovie electrocautery \\
4 & Cuffed Tracheostomy tube ranging in size 7-8 shiley XLT. \\
5 & Portable Headlight/Shoulder roll. \\
6 & Viral filter. \\
7 & Surgical hemostatic agents \\
8 & Standard Full PPE (N95 mask, gown, gloves, face shield/goggles, shoe covers). \\
\hline
\end{tabular}

Table 3. Personnel for Tracheostomy.

Table 4. Tracheostomy Protocol.

\begin{tabular}{ll}
\hline 1 & Preoperative briefing of Tracheostomy protocol by all team members. \\
2 & PPE administration, Sterile gowning, enter negative pressure ICU room. \\
3 & Surgical time out performed. Patient positioning, shoulder roll placed. \\
4 & Patient Pre-oxygenated with $100 \% \mathrm{FiO}_{2}$. \\
5 & Paralytic anesthesia administration. \\
6 & Skin incision, trachea exposed. \\
7 & Ventilator turned off/held. \\
8 & ETT cuff deflated, advanced $2 \mathrm{~cm}$ and ETT cuff re-inflated. \\
9 & Ventilator turned on, given $100 \%$ FiO ${ }_{2}$ for 30 seconds. \\
10 & Ventilator turned off, patient allowed to exhale. \\
11 & ETT clamped. \\
12 & Tracheotomy. \\
13 & ETT visualized by the surgical team and withdrawn by anesthesia. \\
14 & Tracheostomy intubation once distal tip of ETT visualized, cuff over-inflated. \\
15 & Viral filter attached to trach and connected to Ventilator. \\
16 & Vent turned back on, end tidal $\mathrm{CO}_{2}$ used to confirm placement, ETT removed. \\
17 & Skin incision closed and tracheostomy secured. \\
\hline
\end{tabular}

\section{Results}

A total of 17 Tracheostomies were performed at our community hospital who fulfilled our indication criteria (Table 5). The average age was 62 years old, average body mass index (BMI) was 30 and 15 of our 17 patients were male. The most common comorbidities present in our patient population were as follows: 64\% had hypertension (HTN), $47 \%$ had hyperlipidemia (HLD) and 35\% had type 2 diabetes mellitus (DM2). Secondary to COVID-19 patient's high incidence of thrombotic events, 59\% of our patients were actively on therapeutic anticoagulation regimens at time of tracheostomy. Patients had an average endotracheal intubation preoperative time period of 24 (17-35) days. The average duration of tracheostomy which was measured from skin incision until all team members exited the ICU room was 19 (13-31) minutes. The average AP1 was 26 (14-61) seconds and AP2 was 79 (40-166) seconds with the total apneic period time averaging 106 (67-153) seconds which 
did not yield any adverse oxygenation events.

There were minimal complications noted within our study within a mean follow up time of 21 days. These included a minor complication of oozing from the tracheostomy site which required suture ligation and two major complications; tracheostomy revision for displacement on postoperative day 7 (POD) and a left sided pneumothorax which required a chest tube insertion on POD 1. At the time of completion of this manuscript four patients were decannulated, one patient was downsized, tolerating capping trials and eight patients had been discharged to rehabilitation centers. Four patients died as a result of cardiopulmonary effects of COVID-19, unrelated to the tracheostomy procedure, yielding a mortality rate of $23 \%$. No healthcare workers involved with tracheostomy procedure displayed symptoms or documented newly diagnosed COVID-19 positive status.

Table 5. Tracheostomy Patient Data.

\begin{tabular}{|c|c|c|c|c|c|}
\hline Patient & Age & Gender & BMI & Comorbidities & Preop vent setting \\
\hline 1 & 57 & $\mathrm{M}$ & 31 & HTN, DM2 & AC $3050040 \% 5$ \\
\hline 2 & 63 & M & 32 & HLD, GERD & AC $3045040 \% 5$ \\
\hline 3 & 55 & M & 26 & HTN, DM2, HLD, CKD3 & AC $1446030 \% 5$ \\
\hline 4 & 58 & $\mathrm{~F}$ & 28 & HLD, DM2, HTN & AC $1648040 \% 5$ \\
\hline 5 & 41 & M & 24 & None & AC $3440040 \% 5$ \\
\hline 6 & 57 & M & 31 & HTN, HLD & AC $3249040 \% 6$ \\
\hline 7 & 62 & M & 37 & Anemia, Gout, GERD, HLD, HTN, OSA, TIA & AC $1850030 \% 5$ \\
\hline 9 & 75 & M & 37 & HTN, DM2, hypothyroid & AC $1845035 \% 5$ \\
\hline 10 & 78 & $\mathrm{~F}$ & 36 & HTN & AC $2045040 \% 6$ \\
\hline 11 & 62 & M & 34 & OSA & AC $2050040 \% 8$ \\
\hline 12 & 72 & M & 24 & $\mathrm{BPH}$ & AC $3045040 \% 5$ \\
\hline 13 & 58 & M & 27 & HTN, HLD, CAD & AC $1842040 \% 5$ \\
\hline 14 & 69 & M & 33 & HTN, DM2 & AC $3445040 \% 5$ \\
\hline 15 & 54 & M & 27 & Asthma, Fibromyalgia & AC $2636040 \% 8$ \\
\hline 16 & 47 & M & 39 & None & AC $3445040 \% 5$ \\
\hline
\end{tabular}

Table 5. Continued.

\begin{tabular}{|c|c|c|c|c|c|c|}
\hline Patient & Preop Days intubated & AP1 (sec) & AP2 (sec) & Total Apneic period (sec) & Duration (Min) & Tracheostomy Complications \\
\hline 1 & 21 & 40 & 44 & 84 & 25 & None \\
\hline 2 & 22 & 27 & 40 & 67 & 21 & Minimal bleeding \\
\hline 3 & 23 & 31 & 122 & 153 & 15 & LPTHX POD 1 Trach CT placed \\
\hline 4 & 21 & 50 & 51 & 101 & 12 & None \\
\hline 5 & 23 & 18 & 72 & 90 & 31 & Revision for dislodged Trach POD7 \\
\hline 6 & 30 & 15 & 94 & 109 & 19 & None \\
\hline 7 & 23 & 41 & 60 & 101 & 13.00 & None \\
\hline 8 & 20 & 37 & 49 & 86 & 18 & None \\
\hline 9 & 22 & 61 & 44 & 105 & 23 & None \\
\hline 10 & 17 & 15 & 80 & 95 & 14 & None \\
\hline 11 & 35 & 23 & 165 & 188 & 18 & None \\
\hline 12 & 31 & 16 & 67 & 83 & 18 & None \\
\hline 13 & 18 & 14 & 86 & 100 & 18 & None \\
\hline 14 & 21 & 19 & 106 & 125 & 13 & None \\
\hline 15 & 21 & 19 & 75 & 94 & 13 & None \\
\hline 16 & 33 & 15 & 127 & 142 & 25 & None \\
\hline 17 & 29 & 15 & 75 & 90 & 15 & None \\
\hline
\end{tabular}

Ingredients of a Successful Tracheostomy in COVID-19 Patient Population

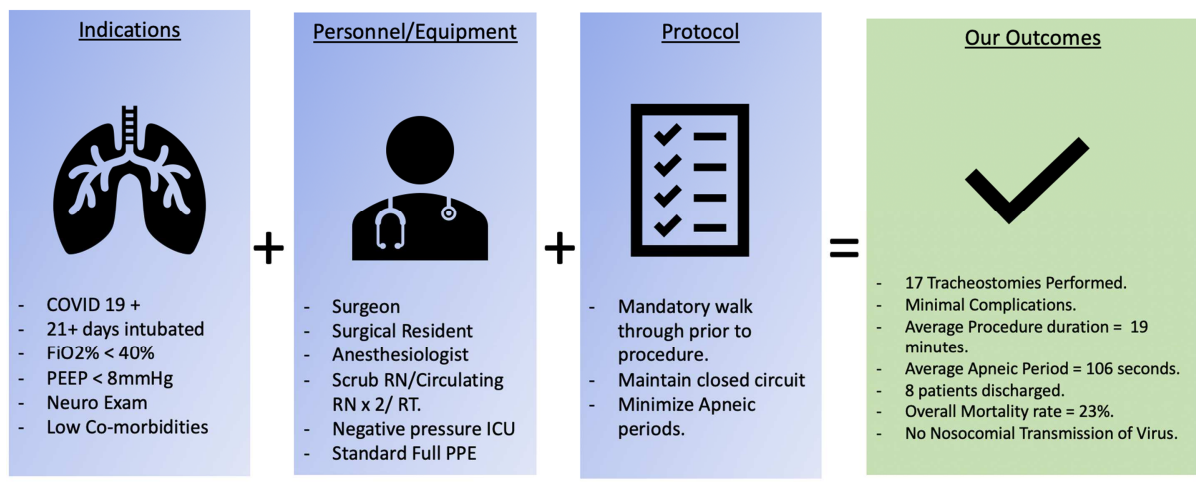

Figure 1. Visual Abstract. 


\section{Discussion}

The COVID-19 pandemic presents unique difficulties when considering performing a tracheostomy for such patients for a variety of reasons. First, the prognosis of this novel disease is not yet fully understood but preliminary mortality rates for those requiring critical care have been estimated as high as $70-80 \%$. This in general is noticeably greater than patients who are critically ill with non-COVID pneumonia. Thus, there is a heightened risk of performing a futile procedure. Second, the duration of detectable viral load and correlation with transmission rates during aerosol generating procedures is not yet specifically known. Therefore, there is an increased risk of infection to the healthcare workers performing a potentially futile procedure. Finally, there is no evidence to support a standardized approach for tracheostomy insertion in terms of minimizing risk of healthcare personnel exposure to airborne droplets. It is not surprising that there remains little literature documenting implementation of high-risk procedures in highly contagious upper airway diseases.

Post-intubation laryngo-tracheal stenosis is a well-known risk of prolonged endotracheal intubation. There is a challenge involved in balancing this complication with the ethical issue of if a tracheostomy should be performed on these patients at all. If so, how to perform the procedure minimizing the risk to healthcare workers providing care is of critical importance. We attempt to mitigate these variables, vetted in a multidisciplinary approach, with a stringent protocol applied to the surgical end.

In our recent history we have not been confronted with a pandemic that COVID-19 has surmounted. Therefore, we must curate innovative strategies to respond to this novel entity. With our proposed tracheostomy protocol, efforts to maintain a closed system and thus limit aerosolized virus to the provider was at utmost importance. The endotracheal tube was advanced prior to tracheotomy in order to decrease incidence of injuring the balloon cuff in the circumstance of tracheostomy failure and need for resumption of a closed system endotracheal airway. We found that a tracheostomy was not more difficult and performed in a timely fashion (averaging 18 minutes in duration) with an experienced provider performing the procedure despite a suboptimal setting and patient positioning. We sought out to calculate and limit apnea periods in this specific patient population as they are hypothesized to have less pulmonary reserve due to their severe COVID-19 pneumonia. The total apneic period during tracheostomy within our study of 106 seconds on average did not yield any observed hypoxic events. We observed a low incidence of postoperative bleeding events even with $59 \%$ of our patient population being restarted on therapeutic anticoagulation on POD 1 for thrombotic COVID-19 sequela. The relatively low postoperative mortality rate compared to the overall mortality rate of intubated COVID-19 patients indicated that our preoperative indications may reliably be used to determine candidacy for Tracheostomy in COVID-19 patients. Furthermore, tracheostomy may offer a benefit for these patients as conventional bronchoscopy and lavage should be avoided, allowing for better suctioning and viral pneumonia clearance. At the time of publication, no nosocomial transmission of virus has been observed in any providers participating in the study confirming the safety of the proposed protocol.

\section{Conclusion}

Surgical airways and tracheostomy care, normally considered routine, carries significant risk of COVID-19 exposure to healthcare providers and the community. This preliminary study indicates that promising outcomes can be achieved with tracheostomy in the COVID-19 patient population. A key feature of our proposed protocol is that it can readily be implemented with the resource's community hospitals have readily available.

The COVID-19 pandemic presented the surgical community with novel quandaries. We recommend a multidisciplinary approach to tracheostomy, which can be performed in this high-risk population if strict indications, equipment, personnel and protocol recommendations are followed. No nosocomial transmission was observed while minimizing apneic periods and overall mortality with this protocol. A larger study of COVID-19 tracheostomy patients with extended follow up is indicated to confirm the long-term efficacy of our proposed protocol. As this pandemic continues to evolve, the healthcare community will continue to be challenged with problematic scenarios. As evidence-based guidelines and recommendations evolve, practices and procedures will need to be continually reevaluated to ensure safety and appropriateness in high-risk populations.

\section{Abbreviations}

$\begin{array}{ll}\text { AC } & \text { Assist Control } \\ \text { AP1 } & \text { Apneic period 1 } \\ \text { AP2 } & \text { Apneic period 2 } \\ \text { CAD } & \text { Coronary Artery Disease } \\ \text { CKD3 } & \text { Chronic Kidney Disease Stage 3 } \\ \text { COVID-19 } & \text { Coronavirus disease 2019 } \\ \text { DM2 } & \text { Diabetes Mellitus 2 } \\ \text { FiO } 2 & \text { Fraction of inhaled of inhaled oxygen } \\ \text { GERD } & \text { Gastroesophageal reflux disease } \\ \mathrm{HLD} & \text { Hyperlipidemia } \\ \mathrm{HTN} & \text { Hypertension } \\ \mathrm{ICU} & \text { Intensive care unit } \\ \mathrm{MERS} & \text { Middle East Respiratory Syndrome } \\ \text { OR } & \text { Operating room } \\ \text { OSA } & \text { Obstructive sleep apnea } \\ \text { PCR } & \text { Polymerase chain reaction } \\ \text { PEEP } & \text { Positive end expiratory pressure }\end{array}$




$\begin{array}{ll}\text { POD } & \text { Postoperative day } \\ \text { PPE } & \text { Personal Protective Equipment } \\ \text { RN } & \text { Registered Nurse } \\ \text { SARS } & \text { Severe Acute Respiratory Syndrome } \\ \text { TIA } & \text { Transient Ischemic attack } \\ \text { XLT } & \text { Extended Length Tracheostomy }\end{array}$

\section{Conflict of Interest}

All the authors do not have any possible conflicts of interest.

\section{Funding Information}

This research did not receive any specific grant from funding agencies in the public, commercial, or not-for-profit sectors.

\section{Informed Consent}

Full detailed written consent was obtained from patient's healthcare proxy for Tracheostomy to be performed with the proposed protocol as well as permission to collect data perioperatively. The protocol was reviewed and approved by a multi-disciplinary team at Huntington Hospital, NY.

\section{Author Contributions}

B. T. is guarantor and has full responsibility over the manuscript. B. T., M. S. and V. A. S. searched literature and wrote the manuscript. B. T., V. A. S., R. Z., R. K. and D. G. contributed substantially to the study design, development of protocol, data analysis and interpretation and reviewed the manuscript. B. T., V. A. S. and D. G. were responsible for performing all procedures described within this manuscript.

\section{Acknowledgements}

Would like to recognize Dr. Chris Papadopoulos, Dr. John Anderson, Dr. Peter Shih, Dr. Robert Herenstein, Dr. Fred DiBlasio, C. Sikorsky, A. Maisch, M. Palma, K. Cunningham in their assistance throughout performing the procedure and perioperative care of these patients.

\section{References}

[1] Worldometer. COVID-19 coronavirus pandemic. June 10, 2020. https://www.worldometers.info/coronavirus/ (accessed June 10, 2020).

[2] Tay JK, Khoo ML, Loh WS. Surgical Considerations for Tracheostomy During the COVID-19 Pandemic: Lessons Learned From the Severe Acute Respiratory Syndrome Outbreak. JAMA Otolaryngol Head Neck Surg. 2020.

[3] Durbin CG. Tracheostomy: why, when, and how?. Respir Care. 2010; 55 (8): 1056-68.

[4] Carlos WG, Dela cruz CS, Cao B, Pasnick S, Jamil S. Novel Wuhan (2019-nCoV) Coronavirus. Am J Respir Crit Care Med. 2020; 201 (4): P7-P8.

[5] Han Y, Yang H. The transmission and diagnosis of 2019 novel coronavirus infection disease (COVID-19): A Chinese perspective. J Med Virol. 2020.

[6] Tien HC, Chughtai T, Jogeklar A, Cooper AB, Brenneman F. Elective and emergency surgery in patients with severe acute respiratory syndrome (SARS). Can J Surg. 2005; 48 (1): 71-4.

[7] Ahmed N, Hare GM, Merkley J, Devlin R, Baker A. Open tracheostomy in a suspect severe acute respiratory syndrome (SARS) patient: brief technical communication. Can J Surg. 2005; 48 (1): 68-71.

[8] Kwan A, Fok WG, Law KI, Lam SH. Tracheostomy in a patient with severe acute respiratory syndrome. $\mathrm{Br} \mathrm{J}$ Anaesth. 2004; 92 (2): 280-2.

[9] Chee VW, Khoo ML, Lee SF, Lai YC, Chin NM. Infection control measures for operative procedures in severe acute respiratory syndrome-related patients. Anesthesiology. 2004; 100 (6): 1394-8.

[10] Jacob T, Walker A, Mantelakis A, Gibbins N, Keane O. A framework for open tracheostomy in COVID-19 patients. Clin Otolaryngol. 2020.

[11] Expert consensus on preventing nosocomial transmission during respiratory care for critically ill patients infected by 2019 novel coronavirus pneumonia]. Zhonghua Jie $\mathrm{He} \mathrm{He} \mathrm{Hu}$ Xi Za Zhi. 2020; 17: E020.

[12] Foster P, Cheung T, Craft P, et al. Novel Approach to Reduce Transmission of COVID-19 During Tracheostomy. J Am Coll Surg. 2020.

[13] Rassekh et al. Tracheostomy in Ventilated Patients with COVID-19. Annals of Surgery. COVID 19 Online resource, 2020 . 